\title{
REPRESENTATION OF FUNCTIONS AS ABSOLUTELY CONVERGENT FOURIER-STIELTJES TRANSFORMS
}

\section{EDWIN HEWITT}

0. Introduction. Let $G$ be a locally compact Abelian group, and $G^{*}$ its group of (continuous) characters. We write $(y, x)$ to indicate the function on $G^{*} \times G$ which is the value of the character $y \in G^{*}$ at the point $x \in G$, or, dually, the value of the character $x \in G$ at the point $y \in G^{*}$. Borel sets in $G$ are taken to be the smallest $\sigma$-algebra of sets containing all closed sets. We consider the set of all regular, countably additive, complex-valued, Borel measures on $G$. (For measuretheoretic terms not defined here, see [4].) For any such measure $\phi$, let $\|\phi\|=\sup \sum_{n=1}^{n}\left|\phi\left(A_{v}\right)\right|$, the supremum being taken over all finite collections $\left\{A_{n}\right\}_{n=1}^{n}$ of Borel subsets of $G$ which are pairwise disjoint and whose union is $G$. We postulate that $\|\phi\|$ be finite. Such measures are called here bounded Radon measures. The measure $\epsilon_{x}$ such that $\epsilon_{x}(A)=1$ or 0 as $A$ does or does not contain the point $x \in G$ is a particularly simple bounded Radon measure.

Let $\mathfrak{S}_{\infty}(G)$ denote the linear space of all continuous complex-valued functions $f$ on $G$ such that for every $\epsilon>0$, there is a compact subset $A$ of $G$ such that $|f(x)|<\epsilon$ for $x \notin A$. It is known [6] that a complex linear functional $L$ on $\mathfrak{S}_{\infty}(G)$ which is bounded in the uniform norm has a unique representation as the integral over $G$ with respect to a bounded Radon measure $\phi$ :

$$
L(f)=\int_{G} f(x) d \phi(x) .
$$

With the representation 0.1 for $L$, there is a natural extension of $L$ (which is by no means a unique bounded extension, if $G$ is noncompact) over the space $\mathbb{S}(G)$ of all bounded continuous complex functions on $G$. For $g \in \mathbb{E}(G)$, we write

$$
L(g)=\int_{G} g(x) d \phi(x) .
$$

In particular, every character $(y, x)$ is $\phi$-integrable, and we can write

$$
\Phi(y)=\int_{G}(y, x) d \phi(x) .
$$

Presented to the Society, December 27, 1952; received by the editors November $12,1952$. 
The function $\Phi(y)$ is called the Fourier-Stieltjes transform of the measure $\phi$. It is easy to see that every Fourier-Stieltjes transform is bounded in absolute value by $\|\phi\|$, and that it is uniformly continuous. It is also known [11, pp. 121-122] that the class of FourierStieltjes transforms is identical with the class of complex linear combinations of continuous positive-definite functions on $G^{*}$. It is natural to examine those groups $G$ for which every bounded, uniformly continuous complex function on $G^{*}$ is a Fourier-Stieltjes transform, since many difficulties in studying measures $\phi$ are obviated when this is the case. We shall show that the only groups for which this holds are finite, so that the impossibility of representing the space of measures $\phi$ as the space of all bounded uniformly continuous functions on $G^{*}$ appears as a necessary concomitant of all Fourier-Stieltjes analysis for infinite groups. A reader of [10] and the present note will be aware of our debt to I. E. Segal, whose paper [10] has suggested the problem here solved and also parts of the proof.

1. Statement of results. We actually obtain a slightly sharper result than announced in $\S 0$. For any locally compact group $H$, let $\mathfrak{U}(H)$ denote the set of continuous almost periodic functions on $H$. Here, where we deal only with Abelian $H$, we characterize $\mathfrak{A}(H)$ as the uniform closure of the space of finite complex linear combinations of continuous characters of $H$ (see e.g. [9, p. 126, Satz 1]). We then have the following:

1.1 ThEOREM. Let $G$ and $G^{*}$ be as in $\$ 0$. If every bounded, uniformly continuous, complex function on $G^{*}$ is a Fourier-Stieltjes transform, then $G$ and $G^{*}$ are finite.

We establish 1.1 by proving the following stronger result:

1.2 Theorem. If $G$ and $G^{*}$ are as in $\$ 0$, and if every function in $\mathfrak{2}\left(G^{*}\right)$ is a Fourier-Stieltjes transform, then $G$ and $G^{*}$ are finite.

1.3 THEOREM. If $G$ is an infinite locally compact Abelian group, then the set of Fourier-Stieltjes transforms which lie in $\mathfrak{A}\left(G^{*}\right)$ is a dense set of first category in $\mathfrak{A}\left(G^{*}\right)$, metrized by the uniform norm.

1.4 Theorem. Let $G$ be a locally compact Abelian group satisfying condition 3.1 below. Let $\phi$ be a bounded Radon measure on G. Let $\left\{x_{n}\right\}_{n=1}^{\infty}$ be the (necessarily countable) set of points in $G$ for which $\phi\left(\left\{x_{n}\right\}\right) \neq 0$. If the measure $\phi-\sum_{n=1}^{\infty} \phi\left(\left\{x_{n}\right\}\right) \epsilon_{x_{n}}$ is not zero, then the Fourier-Stieltjes transform $\int_{G}(y, x) d \phi(x)$ is not almost periodic.

1.5 THEOREM. Let $G$ be a locally compact Abelian group satisfying condition 3.1 below. If the function $h \in \mathfrak{A}\left(G^{*}\right)$ is a Fourier-Stieltjes 
transform, then

$$
h(y)=\sum_{n=1}^{\infty} \alpha_{n}\left(y, x_{n}\right)=\int_{a}(y, x) d \sum_{n=1}^{\infty} \alpha_{n} \epsilon_{x_{n}},
$$

where $\sum\left|\alpha_{n}\right|=\left\|\sum \alpha_{n} \epsilon_{x_{n}}\right\|<\infty$.

We conjecture that the condition 3.1 is not necessary for the validity of Theorems 1.4 and 1.5.

The following sections contain proofs of Theorems 1.2-1.4, in several steps.

2. The case of compact $G^{*}$.

2.1 If $G^{*}$ is compact, then $G$ is discrete $[11$, p. 101], and the bounded Radon measures on $G$ are all of the form $\sum_{n=1}^{\infty} \alpha_{n} \epsilon_{x_{n}}$, where $\sum_{n=1}^{\infty}\left|\alpha_{n}\right|<\infty$. The space of bounded Radon measures on $G$ is thus identifiable with the space $\mathfrak{R}_{1}(G)$. To assert that every continuous almost periodic function on $G^{*}$ is a Fourier-Stieltjes transform is thus equivalent to asserting that $\mathfrak{C}\left(G^{*}\right)$ is mapped onto $\mathbb{R}_{1}(G)$ by the Fourier transformation. This, as Segal shows [10], is equivalent to the assertion that $G$ and $G^{*}$ are finite. Hence Theorem 1.2 is true if $G$ is discrete and $G^{*}$ is compact.

3. Another special case. Suppose that $G$ and $G^{*}$ are as in $\$ 0$ and suppose further that $G^{*}$ has the following property:

3.1 There exists a sequence $\left\{A_{n}\right\}_{n=1}^{\infty}$ of compact subsets of $G^{*}$ such that the von Neumann mean value $M$ (see [9, pp. 31-42]) of an arbitrary continuous almost periodic function $f$ on $G^{*}$ is given by the equality

$$
M(f)=\lim _{n \rightarrow \infty} \frac{1}{\mu\left(A_{n}\right)} \int_{A_{n}} f(y) d y,
$$

where $\mu$ denotes Haar measure on $G^{*}$, suitably normalized, and $d y$ indicates integration with respect to this measure.

3.2 LyubarskiY has proved [8] that every connected $G^{*}$ has this property, while Segal has noted [10] that if $G^{*}$ is generated by a compact neighborhood of the identity, then Lyubarskir's proof is valid.

3.3 A simple argument shows that 3.1 is valid for every countably infinite locally compact Abelian group $L$ all of whose elements have finite order. (Any countable locally compact group is clearly dis-

${ }^{1} \mathrm{Dr}$. H. Helson has kindly pointed out to the writer, subsequent to the submittal of the present paper for publication, that Theorem 1.4 and therefore Theorem 1.5 are in fact true for all locally compact Abelian groups $G$. His proof depends upon a theorem which will shortly appear in Arkiv för Matematik. 
crete.) In fact, it is clear that $L$ is the union of a countable sequence of increasing finite subgroups: $S_{1} \subset S_{2} \subset S_{3} \subset \cdots \subset S_{n} \subset \cdots$, $\cup_{n-1}^{\infty} S_{n}=L$. The mean value of a character $\chi$ of $L$ on a subgroup $S_{k}$ is either 1 or 0 , as $\chi$ is or is not identically 1 on $S_{k}$. If a character is identically 1 on all $S_{k}$, it is certainly the character 1 . Given an almost periodic function $f$ on $L$. a positive number $\epsilon$, and a finite complex linear combination of characters, $\sum_{v-1}^{n} \alpha_{v} \chi_{v}$, such that

$$
\sup _{x \in L}\left|f(x)-\sum_{n=1}^{n} \alpha_{\nu} x_{\nu}(x)\right|<\epsilon,
$$

simply select a subgroup $S_{p}$ such that the mean value of $\chi_{v}$ on $S_{p}$ is the mean value of $\chi_{v}$ on $L(\nu=1, \cdots, n)$. Then plainly the mean value of $f$ on $S_{p}$ is within $\epsilon$ of the true mean value of $f$.

3.4 We note in passing that locally compact groups exist for which 3.1 fails. The group $R_{d}$ formed by the discrete additive real numbers is such a group. Any sequence $\left\{A_{n}\right\}_{n=1}^{\infty}$ of compact subsets of $R_{d}$ consists of finite sets, and the union $\mathrm{U}_{n=1}^{\infty} A_{n}$ is countable. For any countable set, a Hamel basis argument can be applied to show the existence of a character $\chi$ of $R_{d}$ which is not identically 1 on $R_{d}$ but which is identically 1 on the given countable set. Thus $\lim _{n \rightarrow \infty}\left(1 / \mu\left(A_{n}\right)\right) \int_{A_{n}} \chi(y) d y=1$ for this character, but $M(\chi)=0$.

3.5 Theorem. Let $G$ and $G^{*}$ be as in $\$ 0$ and let 3.1 hold. Let $\phi$ be $a$ bounded Radon measure on $G$ such that $\phi(\{e\})=0$, where $e$ is the identity in $G$, and let $\Phi(y)=\int_{G}(y, u) d \phi(u)$. Then

$$
\lim _{n \rightarrow \infty} \frac{1}{\mu\left(A_{n}\right)} \int_{A_{n}} \Phi(y) d y=0 .
$$

Consider the iterated integral

$$
\frac{1}{\mu\left(A_{n}\right)} \int_{A_{n}} \int_{G}(y, u) d \phi(u) d y .
$$

Let $\epsilon$ be a positive real number, and let $U$ be a neighborhood of $e$ in $G$ for which $|\phi|(U)<\epsilon$. (See $[7, \S 1]$, for a definition of $|\phi|$.) Then, applying Fubini's theorem, we have

$$
\begin{aligned}
\frac{1}{\mu\left(A_{n}\right)} \int_{A_{n}} \int_{G}(y, u) d \phi(u) d y= & \frac{1}{\mu\left(A_{n}\right)} \int_{U} \int_{A_{n}}(y, u) d y d \phi(u) \\
& +\frac{1}{\mu\left(A_{n}\right)} \int_{G U_{U^{\prime}}} \int_{A_{n}}(y, u) d y d \phi(u) .
\end{aligned}
$$


The first integral is plainly less than or equal to $|\phi|(U)<\epsilon$. The second integral is evaluated as follows. For fixed $u \in G \cap U^{\prime}$, $\lim _{n \rightarrow \infty}\left(1 / \mu\left(A_{n}\right)\right) \int_{\Lambda_{n}}(y, u) d y=M((y, u))=0$, since $u \neq e$. Furthermore, $\left|\left(1 / \mu\left(A_{n}\right)\right) \int_{A_{n}}(y, u) d y\right| \leqq 1$ for all $n$. Since $G$ has total $\phi$-measure finite, the integrands $\left(1 / \mu\left(A_{n}\right)\right) \int_{\Lambda_{n}}(y, u) d y$ are all bounded in absolute value by a positive function integrable $(\phi)$. Hence we can apply Lebesgue's theorem on dominated convergence, and write

$$
\lim _{n \rightarrow \infty} \frac{1}{\mu\left(A_{n}\right)} \int_{G} \int_{A_{n}}(y, u) d y d \phi(u)=0 .
$$

It follows that

$$
\limsup _{n \rightarrow \infty}\left|\frac{1}{\mu\left(A_{n}\right)} \int_{A_{n}} \int_{G}(y, u) d \phi(u) d y\right|<\epsilon,
$$

and hence 3.5.1 holds.

3.6 TheOREM. If $G$ and $G^{*}$ are as in $\$ 0$ and 3.1 holds, and if $\phi$ is a bounded Radon measure on $G$ not of the form $\sum_{n=1}^{\infty} \alpha_{n} \epsilon_{x_{n}}$, then $\Phi(y)$ is not almost periodic.

Let $\left\{u_{n}\right\}_{n=1}^{\infty}$ be the set of points in $G$ for which $\phi \neq 0$, and let $\psi=\phi-\sum_{n-1}^{\infty} \phi\left(\left\{u_{n}\right\}\right) \epsilon_{u_{n}}$. By hypothesis, $\psi \neq \neq 0$. The function $\sum_{n-1}^{\infty}\left(y, u_{n}\right) \phi\left(\left\{u_{n}\right\}\right)$ on $G^{*}$ being obviously almost periodic, it follows that $\Psi(y)=\int_{G}(y, u) d \psi(y)$ is almost periodic if $\Phi(y)$ is almost periodic. The function $|\Psi(y)|^{2}$ is the Fourier transform of a certain measure, $\psi * \tilde{\psi}$, which can be shown to vanish for all points. Hence, by Theorem 3.5, $M_{y}\left(|\Psi(y)|^{2}\right)=0$, and it follows that $|\Psi(y)|^{2}=|\Psi(y)|$ $=\Psi(y)=0$. The uniqueness theorem for Fourier-Stieltjes transforms $[3$, p. 88 , Théorème 1$]$ shows that $\psi=0$, which is a contradiction; and so the theorem is proved.

3.7 Theorem. Let $G$ and $G^{*}$ be as in $\$ 0$ and let 3.1 hold. Then, if every continuous almost periodic function $h$ on $G^{*}$ is a Fourier-Stieltjes transform, $G$ and $G^{*}$ are finite.

It follows from Theorem 3.6 that $h$ must be of the form $\sum_{n=1}^{\infty} \alpha_{n}\left(y, x_{n}\right)$, where $\sum_{n=1}^{\infty}\left|\alpha_{n}\right|<\infty$, if $h$ is almost periodic and a Fourier-Stieltjes transform. It is known (see for example [1]) that on imbedding $G^{*}$ in a suitable compact group $b G^{*}$, which may be called the Bohr compactification of $G^{*}$, the continuous almost periodic functions on $G^{*}$ become the continuous functions on $b G^{*}$, and von Neumann mean values on $G^{*}$ become Haar integrals on $b G^{*}$. The character group of $b G^{*}$ is the discrete group $G$, written as $G_{d}$. Under our present hypotheses, we find that every continuous function on $b G^{*}$ 
has a Fourier transform in $\mathbb{R}_{1}\left(G_{d}\right)$. Thus the image of $\mathbb{C}\left(b G^{*}\right)$ under Fourier transforms is identical with $\mathfrak{R}_{1}\left(G_{d}\right)$, and consequently the image of $\mathfrak{R}_{1}\left(G_{d}\right)$ is $\left(b G^{*}\right)$. By $\$ 2$, this implies that $b G^{*}$ and $G_{d}$ are finite, hence that $G$ and $G^{*}$ are finite.

Theorems 1.2 and 1.1 are thus established under the hypothesis 3.1. Theorems 1.4 and 1.5 are immediate consequences of the foregoing.

4. The general case. We now aim to reduce the general case to the case treated in $\$ 3$. To this end, we establish a useful fact of some interest on its own account.

4.1 Theorem. Let $L$ be any locally compact Abelian group and let $H$ be a closed subgroup of $L$. Then any continuous almost periodic function on $H$ admits a continuous almost periodic extension over $L$.

The Bohr compactification $b L$ of $L$ can be identified with the group of all characters, continuous or discontinuous, of the group $L^{*}$, which is the group of continuous characters of $L$. Since $H$ is a closed subgroup of $L$, the character group of $H$ can be identified with the quotient group $L^{*} / \Delta$, where $\Delta$ is the group of characters of $L$ which are identically 1 on $H$. The Bohr compactification $b H$ is therefore the group of all characters, continuous or discontinuous, of the group $L^{*} / \Delta$. This is the group of all characters of $L^{*}$ which are constant on cosets modulo $\Delta$. It follows that $b H$ is a compact subgroup of $b L$. Every continuous almost periodic function on $H$ has a unique continuous extension over $b H$. Every continuous complex function on $b H$ has an infinite number of continuous extensions over $b L$ (since $b H$ and $b L$ are compact, and therefore $b L$ is normal and $b H$ is closed in $b L$ ). For every continuous function on $b L$, the contraction of this function to $L$ is almost periodic. This proves the present theorem.

4.2 Theorem. Let $G$ and $G^{*}$ be such that every continuous almost periodic function on $G^{*}$ is a Fourier-Stieltjes transform. If $H$ is a closed subgroup of $G^{*}$, then every continuous almost periodic function on $H$ is a Fourier-Stieltjes transform, of a bounded Radon measure on the character group of $H$.

Let $p(y)$ be a continuous almost periodic function on $H$. By 4.1, there exists a continuous almost periodic function $q(y)$ on $G^{*}$ such that $q(y)=p(y)$ for $y \in H$. By hypothesis, $q(y)=\int_{G}(y, x) d \phi(x)$ for some bounded Radon measure $\phi$ on $G$. This implies that $q(y)=q_{1}(y)$ $-q_{2}(y)+i\left(q_{3}(y)-q_{4}(y)\right)$, where $q_{1}, q_{2}, q_{3}, q_{4}$ are continuous positivedefinite functions on $G^{*}$. These functions, considered on $H$ alone, are clearly still positive-definite and continuous. Hence the function 
$p(y)=p_{1}(y)-p_{2}(y)+i\left(p_{3}(y)-p_{4}(y)\right)$, where $p_{1}, p_{2}, p_{3}, p_{4}$ are continuous positive-definite functions on $H$. It follows from the theorem of Bochner-Weil [11, pp. 121-122] that $p$ is a Fourier-Stieltjes transform.

We can now prove Theorem 1.2. Let $H$ be the closed subgroup of $G^{*}$ generated by a compact neighborhood of the identity. Then, from the hypotheses of Theorem 1.2, and Theorem 4.2, we infer that every continuous almost periodic function on $H$ is a Fourier-Stieltjes transform. From 3.1 and 3.4, we infer that $H$ is finite. This implies that $G^{*}$ is discrete and that every element of $G^{*}$ has finite order. Let $L$ be any countable subgroup of $G^{*}$. Then, by Theorem $4.2, L$ satisfies the hypotheses of Theorem 1.2. But $L$ also enjoys property 3.1 , as is pointed out in 3.3, and therefore is finite. Since any infinite Abelian group has countably infinite subgroups, we see that $G^{*}$, and hence $G$, must be finite. Theorem 1.3 follows at once from Theorem 1.2 and a theorem of Banach $[2$, p. 38 , Théorème 3$]$.

5. Specific constructions. Actually to exhibit continuous bounded functions on $G^{*}$ which are not Fourier-Stieltjes transforms is somewhat laborious. We give three simple examples.

5.1 Consider the circle group $T$ and its character group, the integers $N$. The function $h$ on $N$ such that $h(k)=1$ for $k>0$ and 0 for $k \leqq 0$ is not a Fourier-Stieltjes transform. If it were, then the functional $H\left(\sum_{k=-n}^{n} \alpha_{k} e^{i k x}\right)=\sum_{k=-n}^{n} \alpha_{k} h(k)=\sum_{k=1}^{n} \alpha_{k}$ would be bounded in absolute value for all trigonometric polynomials $\sum_{k=-n}^{n} \alpha_{k} e^{i k x}$ which have a common bound. Choosing $\alpha_{k}=1 / 2 i k(k= \pm 1, \pm 2, \cdots, \pm n)$ and $\alpha_{k}=0$ otherwise, we have $\sum_{k=-n}^{n} \alpha_{k} e^{i k x}=\sum_{k=1}^{n}(1 / k) \sin k x$, which is bounded in absolute value for all $n$ (see, e.g., [5, p. 29]). However $\sum_{k=-n}^{n} \alpha_{k} h(k)=\sum_{k=1}^{n} 1 / k$, which is unbounded.

5.2 By similar reasoning, if $f(t)$ is any continuous bounded function on the line $R$ such that $f(t)=1$ for $t>A$ and $f(t)=0$ for $t<-A$, for some $A>0$, then $f(t)$ cannot be written as $\int_{-\infty}^{\infty} e^{i t x} d \phi(x)$ for any bounded Radon measure $\phi$ on the line.

5.3 Let $p(t)$ be a continuous periodic function on the line whose Fourier series does not converge absolutely. Then $p$ is a continuous almost periodic function which cannot be written as $\int_{-\infty}^{\infty} e^{i t x} d \phi(x)$, as Theorem 1.5 shows.

\section{BIBLIOGRAPHY}

1. Hirotada Anzai and Shizuo Kakutani, Bohr compactifications of a locally compact Abelian group, I and II, Proc. Imp. Acad. Tokyo vol. 19 (1943) pp. 476-480 and 533539.

2. Stefan Banach, Theorie des operations linéaires, Monografje Matematyczne, Warsaw, 1932.

3. Henri Cartan and Roger Godement, Theorie de la dualité et analyse harmonique 
dans les groupes abeliens localement compacts, Ann. Ecole Norm. (3) vol. 64 (1947) pp. 79-99.

4. Paul R. Halmos, Measure theory, New York, Van Nostrand, 1950.

5. G. H. Hardy and W W. Rogosinski, Fourier series, Cambridge Tracts in Mathematics and Mathematical Physics, no. 38, Cambridge University Press, 1950.

6. Edwin Hewitt, Integration on locally compact spaces I, University of Washington Publications in Mathematics, vol. 3 (1952) pp. 71-75.

7. - Remarks on the inversion of Fourier-Stieltjes transforms, Ann. of Math. (2) vol. 57 (1953) pp. 458-473.

8. G. Ya. Lyubarskil, On the integration in the mean of almost periodic functions on topological groups, Uspehi Mat. Nauk N.S. vol. 3 (1948) pp. 195-201.

9. Wilhelm Maak, Fastperiodische Funktionen, Grundlehren der Mathematischen Wissenschaften, vol. 61, Berlin, Springer, 1950.

10. Irving E. Segal, The class of functions which are absolutely convergent Fourier transforms, Acta Sci. Math. Szeged vol. 12 (1950) pp. 157-161.

11. Andre Weil, L'intégration dans les groupes topologiques et ses applications, Paris, Hermann, 1940.

THE UNIVERSITY OF WASHINGTON 